\title{
Relación entre celo-inseminación con semen sexado y porcentaje de preñez en vaquillonas Holstein
}

\author{
Marini, P.R.; Galassi, I. \\ Cátedra de Producción de Bovinos Lecheros, Facultad de Ciencias Veterinarias, \\ Universidad Nacional de Rosario. Ovidio Lagos y Ruta 33, (2170) Casilda, \\ Provincia de Santa Fe (Argentina). E-mail: pmarini@fveter.unr.edu.ar.
}

\begin{abstract}
Resumen
Marini, P.R.; Galassi, I.: Relación entre celo-inseminación con semen sexado y porcentaje de preñez en vaquillonas Holstein. Rev. vet. 22: 1, 52-54, 2011. El objetivo del presente trabajo fue estudiar la relación entre la duración del período transcurrido entre la detección del celo y la inseminación con semen sexado y el porcentaje de preñez en vaquillonas Holstein y -en caso de existir- si dicha relación es afectada por el origen del semen utilizado (efecto toro). En un estudio de carácter retrospectivo se evaluó información recolectada durante el año 2009 sobre 607 períodos celos-servicios correspondientes a 607 vaquillonas Holstein. Para los servicios efectuados durante el período analizado se utilizó semen de cinco toros. Se registraron observaciones de celos e inseminaciones durante cuatro veces por día. En función de esta información las vaquillonas se categorizaron de acuerdo al lapso transcurrido (horas) entre la detección del celo y su inseminación, en cuatro grupos: 9:00 a 10:59, 11:00 a 12:59, 13:00 a 14:59 y 15:00 a 17:00 horas. No hubo diferencias significativas $(p>0,05)$ entre los distintos grupos y el porcentaje de preñez. Por otro lado, el efecto toro provocó diferencias significativas en el porcentaje de preñez $(\mathrm{p}<0.05)$. Se concluye que en las inseminaciones efectuadas entre los lapsos estudiados después de la iniciación del celo no se producen repercusiones negativas sobre el porcentaje de preñez, pero sí con el tipo de toro utilizado.
\end{abstract}

Palabras clave: vaquillona lechera, semen sexado, intervalo celo-inseminación, porcentaje de preñez.

\begin{abstract}
Marini, P.R.; Galassi, I.: Relationship between estrus-insemination with sex-sorted semen and conception rate in Holstein dairy heifers. Rev. vet. 22: 1, 52-54, 2011. The aim of this work was the study of the relationship between the length of the period passed from the estrus detection to the insemination with sex-sorted semen and the conception rate in dairy heifers, and whether this relationship (if there is one) is affected by the origin of the semen (bull effect). In a retrospective study, information gathered during 2009 on 607 estrus-service periods, corresponding to 607 Holstein heifers, was assessed. For the services carried out during the period under analysis, semen from five bulls was used. Observations on estrus and inseminations were registered four times a day. Based on this information, heifers were sorted out according to the interval (in hours) between the estrus detection and their insemination in four groups: 9:00-10:59, 11:00-12:59, 13:00-14:59 and 15:00-17:00 hours. There were no significant differences $(\mathrm{p}>0.05)$ between the different groups and the conception rate. The bull effect did mark significant differences $(p<0.05)$ within the conception rate. It is concluded that no negative impact was observed on the conception rate in inseminations made among the studied intervals after the estrus initiation in dairy heifers, but significative differences was observed when considering the type of bull.
\end{abstract}

Key words: dairy heifer, sex-sorted semen, estrus-insemination interval, conception rate

\section{INTRODUCCIÓN}

El sexado del semen es una tecnología de creciente utilización y máximo interés en los programas de inseminación artificial (IA) del ganado productor de leche y carne en nuestro país. Sus efectos sobre la tasa

Recibido: 18 marzo 2011 / Aceptado: 29 abril 2011 de parición de hembras modificarían positivamente los porcentajes de reposición y los costos de crianza de machos. Su utilización en programas de inseminación a celo detectado demuestra porcentajes de preñez promedio que oscilan entre 50 y $55 \%$ en vaquillonas $1,8,9$, ${ }^{10}$. La principal limitación de la técnica se restringe a las bajas tasas de concepción obtenidas en vacas, lo que limita su uso casi exclusivamente a las vaquillonas. Sin 
embargo, también en esta categoría los resultados son muy variables y sus causas, múltiples. El número de espermatozoides por pajuela, la calidad del semen, el sitio de deposición del semen y el momento de la IA en relación al estro, afectan la probabilidad de preñez después de la IA ya sea convencional o con semen sexado ${ }^{3,13}$.

Los espermatozoides obtenidos inmediatamente tras la eyaculación no poseen las condiciones necesarias para poder penetrar las cubiertas del ovocito e iniciar los procesos de fusión gamética, sino que requieren un proceso de capacitación que se lleva a cabo en el tracto genital de la hembra durante su recorrido hacia los oviductos. Si la siembra se realiza con semen congelado, la sobrevida de los espermatozoides se acorta a un período que oscila entre las 12 y las 24 horas dependiendo de la fertilidad y calidad espermática. Debido a esta última razón es que se acostumbra atrasar el momento de la siembra con respecto al comienzo del celo en un intento de asegurar la presencia de espermatozoides viables en el momento de la ovulación.

Tales argumentos fisiológicos fundamentan el sistema "am/pm" de detección de celo e IA desarrollado a partir de evidencia que indican que el momento óptimo para el servicio se presenta entre 6 y 12 horas después del inicio del celo ${ }^{14}$. De acuerdo con este criterio aquellas vacas o vaquillonas que comienzan a estar receptivas durante la mañana deben ser inseminadas al caer la tarde y aquellas a las que se observa receptivas al final de la tarde o por la noche deben ser inseminadas durante la mañana del día siguiente.

En nuestro país existe consenso generalizado acerca del tiempo que debe transcurrir entre la detección del celo y la IA ${ }^{11,12}$. Pese a ello, y aunque en la mayoría de los establecimientos lecheros este lapso es considerado óptimo para llevar a cabo la IA luego de la detección del celo, no siempre se lo respeta de manera tal que, en ocasiones, las vacas y vaquillonas se inseminan antes o después del intervalo mencionado.

En un trabajo anterior realizado con vacas Holando Argentino, se observó que el criterio generalizado por el cual la IA debe efectuarse entre 12 y 24 horas después de la iniciación del celo, podría ser flexibilizado (adelantado) en función de necesidades puntuales sin que sean esperables repercusiones negativas sobre el porcentaje de preñez ${ }^{7}$.

El objetivo del presente trabajo fue estudiar la relación entre la duración del período transcurrido entre la detección del celo y la IA con semen sexado y el porcentaje de preñez en vaquillonas Holstein y -en caso de existir- si dicha relación es afectada por el origen del semen utilizado (efecto toro).

\section{MATERIAL Y MÉTODOS}

En un estudio de carácter retrospectivo se evaluó información recolectada durante los meses de mayo y octubre del año 2009, sobre 607 períodos celos-servicios correspondientes a 607 vaquillonas Holstein provenientes de un establecimiento ubicado en la cuenca
Tabla 1. Lapso transcurrido entre la detección de celo-inseminación (horas) y porcentaje de preñez.

\begin{tabular}{lccc}
\hline $\begin{array}{l}\text { horas } \\
\text { al servicio }\end{array}$ & servicios & $\begin{array}{c}\text { vaquillonas } \\
\text { preñadas }\end{array}$ & $\begin{array}{c}\% \text { de } \\
\text { preñez }\end{array}$ \\
\hline 9:00-10:59 & 126 & 53 & 42 \\
11:00-12:59 & 115 & 56 & 49 \\
13:00-14:59 & 275 & 130 & 47 \\
15:00-17:00 & 91 & 43 & 47 \\
\hline
\end{tabular}

Tabla 2. Relación entre el toro y el porcentaje de preñez.

\begin{tabular}{lccc}
\hline $\begin{array}{l}\mathrm{N}^{\mathrm{o}} \mathrm{de} \\
\text { toro }\end{array}$ & servicios & $\begin{array}{c}\text { vaquillonas } \\
\text { preñadas }\end{array}$ & $\begin{array}{c}\% \mathrm{de} \\
\text { preñez }\end{array}$ \\
\hline 1 & 149 & 82 & 55 \\
2 & 67 & 27 & 40 \\
3 & 224 & 108 & 48 \\
4 & 34 & 15 & 44 \\
5 & 133 & 50 & 38 \\
\hline
\end{tabular}

este de la Provincia de Córdoba. Los animales eran mantenidos bajo un régimen de alimentación basado en el pastoreo de praderas de alfalfa con suplementación energética de grano de maíz. Las vaquillonas en estudio pesaron en promedio $350 \pm 10 \mathrm{~kg}$ y su edad promedio era de $18 \pm 2$ meses. Para los servicios efectuados durante el período analizado se utilizó semen de cinco toros.

Los datos reproductivos utilizados provienen de los controles periódicos efectuados por el veterinario asesor del establecimiento e incluyen el registro tanto de la hora exacta de detección de celo como de la hora exacta de la IA. En todos los casos los celos fueron detectados por un empleado encargado de dicha tarea y entrenado por el veterinario. Se registraron observaciones de celos e inseminaciones durante cuatro veces por día. En función de esta información las vaquillonas se categorizaron de acuerdo al lapso en horas transcurrido entre la detección del celo y su inseminación, en cuatro grupos: 9:00-10:59 horas, 11:00 a 12:59 horas, 13:00 a 14:59 horas y de 15:00 a 17:00 horas.

La proporción observada de hembras preñadas en cada período, bajo la hipótesis que la probabilidad de preñez de las vaquillonas es la misma en todos ellos se analizó, con una prueba de bondad de ajuste extrínseca a los datos basada en la estadística Chi-cuadrado. La relación entre el período transcurrido (en horas) desde la detección de celo hasta la IA y la proporción de vaquillonas preñadas sobre el total de inseminadas se evaluó, dentro de los toros, con una prueba de homogeneidad basada en la estadística Chi-cuadrado.

\section{RESULTADOS}

En la Tabla 1 se muestra que no hubo diferencias significativas entre los distintos grupos $\mathrm{p}=0,72\left(\mathrm{X}^{2}\right.$ $=1,306$ ) y el porcentaje de preñez, aunque se observó un corte entre el primer grupo y los tres restantes.

En la Tabla 2 se exhiben las comparaciones que fueron estadísticamente significativas $\mathrm{p}=0,0408\left(\mathrm{X}^{2}\right.$ 
$=9,984)$, para el efecto porcentaje de preñez dentro de toro.

\section{DISCUSIÓN}

Los porcentajes de preñez encontrados en las vaquillonas bajo ensayo coinciden con lo reportado en trabajos previos relacionados con la fertilidad de las vacas $\mathrm{y} / \mathrm{o}$ vaquillonas lecheras inseminadas con semen sexado ${ }^{2,10}$. Los porcentajes de preñez encontrados en las vaquillonas bajo ensayo coinciden con lo reportado en trabajos previos 2,10 , en relación a la fertilidad de vaquillonas lecheras inseminadas con semen sexado.

Dentro de los límites de los lapsos estudiados $(9$ a 17 horas), la efectividad de la inseminación artificial no se vería afectada por el tiempo transcurrido desde la detección del celo-servicio y los índices de preñez en cada uno de los cuatro lapsos, que muestran un comportamiento homogéneo, en coincidencia con trabajos previos ${ }^{10}$ pero en disidencia con investigadores que no emplearon semen sexado, quienes reportaron una mejor tasa de concepción entre las 4 y 16 horas de comenzado el celo ${ }^{4}$. En experiencias sobre vaquillonas Holstein usando semen sexado se comunicaron más altas tasas de preñez entre las 16 y 24 horas de iniciado el celo, con respecto al otro grupo de 12 a 16 horas de iniciado el celo ${ }^{5}$.

La fertilidad de los toros puede afectar el porcentaje de preñez como consecuencia de diferencias en la tasa de supervivencia espermática en el tracto reproductivo femenino, así como en el tiempo requerido para la capacitación y el transporte de los espermatozoides hasta el lugar de la fecundación. A pesar de que en este trabajo los toros no fueron categorizados a priori en grupos de diferente fertilidad, los resultados demuestran el efecto de la procedencia del semen utilizado (toro) sobre el porcentaje de preñez. Es evidente que se necesitan más estudios para evaluar las respuestas individuales de los toros con semen sexado.

A pesar del clásico concepto que la IA debería efectuarse entre 12 y 24 horas después de la iniciación del celo, existen trabajos que demuestran que modificando el rango de horas (6-18 horas) no se registran repercusiones negativas sobre el porcentaje de preñez, en contraposición con lo recomendado ya a mediados del siglo pasado, que para maximizar la tasa de concepción las vacas debían ser inseminadas en la segunda mitad del estro ${ }^{6,10}$.

Se concluye que en las inseminaciones efectuadas entre las 09:00 y 17:00 horas después de la iniciación del celo en vaquillonas lecheras no se producen repercusiones negativas sobre el porcentaje de preñez, pero sí con el tipo de toro utilizado.

\section{REFERENCIAS}

1. Brogliatti G, Cerrate H, Caballero J, Cataneo L, Cantarelli A, Feula P, Dalla Lasta M, Ferre L, Panarace M, Medina M. 2002. Pregnancy rates and first born calves by artificial insemination using sexed semen in Argentina. Theriogenology 57: 369.

2. Cutaia LE, Veneranda G, Bó G. 2007. Semen sexado: una herramienta tecnológica para el tambo. Producir XXI (Buenos Aires) 15: 52-57.

3. Dalton JC, Nadir S, Bame JH, Noftsinger M, Nebel RL, Saacke RG. 2001. Effect of time of insemination on number of accessory sperm, fertilization rate, and embryo quality in nonlactating dairy cattle. J Dairy Sci 84: 2413-2418 .

4. Dransfield MB, Nebel R, Pearson RE, Warnick LD. 1998. Timing of insemination for dairy cows identified in estrus by a radiotelemetric estrus detection system. $J$ Dairy Sci 81: 1874-1882.

5. Sá Filhoa MF, Ayresa H, Ferreira RM, Nichia N, Fosadob M, Campos EP, Barusellia PS. 2010. Strategies to improve pregnancy per insemination using sex-sorted semen in dairy heifers detected in estrus. Theriogenology 74: $1636-1642$

6. Gwazdauskas FC, Whittier WD, Vinson WE, Pearson RE. 1986 Evaluation of reproductive efficiency of dairy cattle with emphasis on timing of breeding. J Dairy Sci 69 : 290-297.

7. Marini PR, Galassi I, Di Masso JR. 2010. Relación entre lapso detección del celo-inseminación y el porcentaje de preñez en vacas lecheras. InVet 12: 69-73.

8. Medina M, Cerrate H, Caballero J. 2001. Utilización de GnRH en la inseminación artificial con semen sexado en vaquillonas. Material del curso de posgrado, Instituto de Reproducción Animal de Córdoba (IRAC), Argentina.

9. Medina M, Cattaneo L, Caballero J, Panarace M, Ferré L, Dalla Lasta M. 2002. Semen bovino sexado y congelado en Argentina: resultados de su utilización en programas de inseminación artificial, transferencia de embriones y fertilización in vitro. Revista Taurus (Buenos Aires) 4: 13.

10. Schenk JL, Seidel GE. 2008. Timed insemination of heifers with sexed semen. Reprod Fert Develop 20: 214.

11. Saacke RG, Nadir S, Dalton JC, Bame JH, DeJarnette JM, Degelos S, Nebel RL. 1994. Accessory sperm evaluation and bull fertility: an update. Proceedings $15^{\text {th }}$ Tech Conf Artif Insem \& Reprod (Columbia, USA), p. 57-67.

12. Saacke RG, Dalton JC, Nadir S. Nebel RL, Bame JH. 2000. Relationship of seminal traits and insemination time to fertilization rate and embryo quality. Anim Reprod Sci 60-61: 663-677.

13. Saacke RG. 2008. Insemination factors related to timed AI in cattle. Theriogenology 70: 479-484.

14. Thibier M, Wagner HG. 2000. World statistics for artificial insemination in cattle. Proceedings 14th Internat Congress Anim Reprod (ICAR), Upsala (Suecia) 2: 76. 